\title{
A Modified Gas-Kinetic Scheme for Turbulent Flow
}

\author{
Marcello Righi* \\ School of Engineering, Zurich University of Applied Sciences, Technikumstrasse 9, \\ 8401 Winterthur, Switzerland.
}

Received 14 August 2013; Accepted (in revised version) 2 December 2013

Communicated by Kun Xu

Available online 16 April 2014

\begin{abstract}
The implementation of a turbulent gas-kinetic scheme into a finite-volume RANS solver is put forward, with two turbulent quantities, kinetic energy and dissipation, supplied by an allied turbulence model. This paper shows a number of numerical simulations of flow cases including an interaction between a shock wave and a turbulent boundary layer, where the shock-turbulent boundary layer is captured in a much more convincing way than it normally is by conventional schemes based on the Navier-Stokes equations. In the gas-kinetic scheme, the modeling of turbulence is part of the numerical scheme, which adjusts as a function of the ratio of resolved to unresolved scales of motion. In so doing, the turbulent stress tensor is not constrained into a linear relation with the strain rate. Instead it is modeled on the basis of the analogy between particles and eddies, without any assumptions on the type of turbulence or flow class. Conventional schemes lack multiscale mechanisms: the ratio of unresolved to resolved scales - very much like a degree of rarefaction - is not taken into account even if it may grow to non-negligible values in flow regions such as shocklayers. It is precisely in these flow regions, that the turbulent gas-kinetic scheme seems to provide more accurate predictions than conventional schemes.
\end{abstract}

PACS: 47.27.em, 47.27.nb, 47.32.Ff, 47.40.Hg, 47.40.Nm, 47.45.Ab, 47.85.Gj, 47.85.ld

Key words: Gas kinetic theory, turbulence modeling, compressible flow, shock boundary layer interaction.

\section{Introduction}

Turbulence is a multiscale problem: the challenge lies in either capturing all the scales of motion as is done in the DNS approach or modeling the unresolved scales of motion. Most of the turbulence models, in either RANS or LES, still rely on the idea of eddy viscosity - developed in analogy with the kinetic theory of gases. Over a century after its

*Corresponding author. Email address: marcello.righi@zhaw .ch (M. Righi) 
introduction, its drawbacks are well known and accepted [22]. From the kinetic point of view, the most relevant disadvantage remains the lack of a clear scale separation between resolved and unresolved scales of motion. In rarefied gas dynamics, the ratio of unresolved to resolved scales is used to assess the degree of rarefaction and when it reaches a non-negligible value, the validity of the Navier-Stokes set of equations is questioned. In the continuum regime, whenever turbulence is modeled, the degree of rarefaction is also influenced by unresolved turbulent fluctuations and may make the Navier-Stokes approach unsuitable.

Unlike Navier-Stokes schemes, numerical schemes developed on the basis of the Boltzmann equation dispose of "multiscale" mechanisms, i.e. they can adjust to the changing size of unresolved fluctuations. Among these approaches, one may recall the Lattice Boltzmann Method (LBM) and gas-kinetic schemes. The studies by Chen et al. $[5,6]$ have investigated turbulence modeling in the LBM: the present study exploits the findings in $[5,6]$ in order to explore turbulence modeling following the RANS approach with a gas-kinetic scheme. A well-validated gas-kinetic scheme [32], is adapted to the simulation of turbulence following the RANS approach; it does not propose a novel turbulence model nor a new modeling technique. Instead, a turbulent relaxation time is derived from the turbulent quantities provided by a standard two-equation turbulence model. The analysis of the resulting turbulent scheme shows that the turbulent stress tensor is corrected as a function of the degree of rarefaction, which may assume "transitional" values in shocklayers. The results of numerical experiments, based on different examples of shock-turbulent boundary layer interaction, confirm that the gas-kinetic scheme has the ability to do better than conventional schemes.

A number of gas-kinetic schemes have been developed over the latest 20 years [7, $19,20,32,35]$ with the aim to achieve a physically more consistent mathematical model of fluid mechanics. Gas-kinetic schemes are invariably more accurate than conventional schemes, and might be able to resolve shock-layers. Besides, they are more suitable to high-order reconstruction $[17,34,36]$ and may be used as a platform to investigate rarefied flow $[18,33]$.

The most evident characteristics of these schemes concerns the modeling of collisions. Whereas in conventional schemes, collisions are modeled by a viscosity and assuming a linear stress - strain relation, gas kinetic schemes use a relaxation time - related to collision frequency and hence to viscosity. The BGK model, depending on the scheme parameters, generates a number of different contributions: a stress tensor proportional to viscosity and strain rate, but also a number of non-linear correction terms, related to the local degree of rarefaction and the macroscopic gradients [20,32]. These correction terms represent the kinetic effects that affect the transport properties of the flow, as soon as the degree of rarefaction becomes significant. In most of the flows of engineering and scientific interest, rarefaction may become significant only in shocklayers; however, even in the presence of shocks, conventional schemes do not correct advection: the solution of the Riemann problem is unaffected by the viscosity of the flow.

The numerical experiments proposed in this study address in particular the inter- 
action between shock and turbulent boundary layer. Due to the kinetic scale physics, the gas-kinetic scheme disposes of a much better capacity to capture shocks. Therefore, the control of particle collision time is equivalent to the adaptation of different scale flow modeling. Conventional schemes must rely entirely on artificial dissipation to stabilize shocks, which overwhelms molecular and eddy viscosity, whereas the turbulent gas-kinetic scheme remains physically consistent between the two limiting conditions of smooth flow and shock: the gas-kinetic scheme provides instead a smooth transition from kinetic scale to hydrodynamic scale modeling, where the transition is controlled through the variation of the ratio of the time step to the particle collision time. Remarkably, the turbulent stress tensor may become non-linearly related to strain rate tensor, as the non-linear correction terms are activated by the scheme. The linear turbulent stress tensor is considered one of the most important limiting factors in RANS modeling [22]; in conventional schemes, the attempts to generalize the turbulent stress tensor into a Taylor expansion of the strain rate faces the difficulty to find values to all numerical parameters which provide good performance with many flow classes.

This paper is structured as follows. The derivation of the standard, gas-kinetic scheme for laminar flow and the modification into the turbulent gas-kinetic scheme are presented in Section 2. Section 3 is dedicated to numerical experiments. Conclusions are presented in Section 4.

\section{A gas-kinetic scheme for turbulent flow}

\subsection{Derivation of the gas-kinetic scheme}

\subsubsection{BGK model, derivation of Euler and Navier-Stokes equations}

The kernel of a gas-kinetic scheme consists in modeling the fluxes of the conservative variables across computational cells on the basis of the Boltzmann equation, instead of the Navier-Stokes equations. Following [32] the Boltzmann equation is replaced by the BGK Model [2]:

$$
\frac{\partial f}{\partial t}+(v \cdot \nabla) f=\frac{f^{e q}-f}{\tau}
$$

where the relaxation time $\tau$ is related to the frequency of collisions and $f^{e q}$ is a MaxwellBoltzmann distribution function, describing a gas in thermodynamic equilibrium:

$$
f^{e q}=\rho\left(\frac{\lambda}{\pi}\right)^{\frac{N+2}{N}} \exp \left[-\lambda\left(\left(u_{i}-v_{i}\right)^{2}+\xi^{2}\right)\right]
$$

In Eqs. (2.1) and (2.2), where summation convention holds, and in the rest of this section, the macroscopic variables density, velocity and total energy are indicated with $\rho$, $v$ and $E$; the microscopic variables are velocity $u$ and the $N$ effective degrees of freedom of the gas molecules $\xi$. The quantity of effective degrees of freedom is $N=(5-3 \gamma) /(\gamma-1)+$ 
1 , where $\gamma$ is the specific heat ratio. Moreover, $\lambda=m /(2 k T)=\rho / 2 p, m$ is the molecular mass, $k$ is the Boltzmann constant, and $T$ is temperature.

The conservative variables $w=\left[\rho \rho v_{1} \rho v_{2} \rho v_{3} \rho E\right]^{T}$ can be recovered by taking moments of the distribution function:

$$
w=\int \psi f d \Xi
$$

where the elementary volume in phase space is $d \Xi=d u_{1} d u_{2} d u_{3} d \xi$ and:

$$
\psi=\left[\begin{array}{lllll}
1 & v_{1} & v_{2} & v_{3} & \frac{1}{2}\left(u_{i}{ }^{2}+\xi^{2}\right)
\end{array}\right]^{T} .
$$

In order to be practically used in the development of a numerical scheme Eq. (2.1) is expanded according to the Chapman-Enskog method [4,31]; by introducing the nondimensional quantity $\epsilon=\tau / \widehat{\tau}$, where $\widehat{\tau}$ is a reference time scale, Eq. (2.1) is re-written in the form:

$$
f=f^{e q}-\epsilon \widehat{\tau} D f
$$

with $D f=\partial f / \partial t+(u \cdot \nabla) f$. By substituting Eq. (2.5) into the right hand side of the same equation, one obtains:

$$
f=f^{e q}-\epsilon \widehat{\tau} D f^{e q}+\epsilon^{2} \widehat{\tau} D\left(\widehat{\tau} D f^{e q}\right)+\cdots .
$$

The Euler and Navier-Stokes equations can be obtained by taking moments of Eq. (2.6):

$$
\int f d \Xi=\int\left(f^{e q}-\epsilon \widehat{\tau} D f^{e q}+\epsilon^{2} \widehat{\tau} D\left(\widehat{\tau} D f^{e q}\right)+\cdots\right) d \Xi .
$$

Assuming that $\epsilon$ is a small quantity, the expansion in Eq. (2.7) can be truncated. It can be demonstrated (the complete derivation can be found in Cercignani [4] and $\mathrm{Xu}$ [31]) that Eq. (2.7) corresponds to the Euler equations if the terms $\mathcal{O}(\epsilon)$ are dropped, and to the Navier-Stokes equations if the terms $\mathcal{O}\left(\epsilon^{2}\right)$ are dropped. The conditions to recover the Navier-Stokes equations for a diatomic gas are:

- the relation between molecular viscosity and relaxation time must be:

$$
\mu=\tau / p
$$

(bulk viscosity is $2 N /(3 K+9) \mu)$;

- $\operatorname{Pr}=\mu C_{p} / \kappa=1$, this being a known drawback of the BGK model.

Distribution functions at Euler and Navier-Stokes level are therefore:

$$
\begin{aligned}
& f^{\text {Euler }}=f^{e q}, \\
& f^{N S}=f^{e q}-\epsilon \widehat{\tau} D f^{e q} .
\end{aligned}
$$




\subsubsection{Model of the flow at cells interface}

At each interface, at the beginning of a time step, a function $f$ is introduced as a solution to the Boltzmann equation, with initial conditions consistent with the gas states at both sides of the interface. A closed-form solution of the BGK equation in a time interval $[0, t]$ is given by the integral form presented by Kogan [16] and is used to evaluate $f$ :

$$
\begin{gathered}
f^{B G K}\left(x_{1}, x_{2}, x_{3}, t, u_{1}, u_{2}, u_{3}, \xi\right)=\frac{1}{\tau} \int_{o}^{t} f^{e q}\left(x_{1}^{\prime}, x_{2}^{\prime}, x_{3}^{\prime}, t, u_{1}, u_{2}, u_{3}, \xi\right) e^{-\left(t-t^{\prime}\right) / \tau} d t^{\prime} \\
+e^{-t / \tau} f_{0}\left(x_{1}-u_{1} t, x_{2}-u_{2} t, x_{3}-u_{3} t\right),
\end{gathered}
$$

where $x_{1}^{\prime}=x_{1}-u_{1}\left(t-t^{\prime}\right), x_{2}^{\prime}=x_{2}-u_{2}\left(t-t^{\prime}\right), x_{3}^{\prime}=x_{3}-u_{3}\left(t-t^{\prime}\right)$.

For the sake of clarity, in the rest of this section, the interface is assumed to be normal to $x_{1}$ which is indicated with the symbol $x$ to reduce the number of indexes, the microscopic velocity $u_{1}$ is indicated with $u$. The left and right states of the gas are indicated with the suffixes $(l)$ and $(r)$. A third, fictitious gas state representing the gas at the interface is indicated with the suffix $(c)$. The left and right values of the conservative variables $w_{(l)}, w_{(r)}$ and their gradients $w_{(l) / x^{\prime}}, w_{(r) / x}$ are obtained from a standard reconstruction scheme and limiting process (e.g. MUSCL/TVD, ENO, WENO). On both sides a Maxwell-Boltzmann distribution is defined, from $w_{(l)}$ and $w_{(r)}$ :

$$
\begin{aligned}
& f_{(l)}^{e q}=\rho_{(l)}\left(\frac{\lambda_{(l)}}{\pi}\right)^{\frac{N+2}{N}} \exp \left[-\lambda_{(l)}\left(\left(u_{i}-v_{(l)}\right)^{2}+\xi^{2}\right)\right], \\
& f_{(r)}^{e q}=\rho_{(r)}\left(\frac{\lambda_{(r)}}{\pi}\right)^{\frac{N+2}{N}} \exp \left[-\lambda_{(r)}\left(\left(u_{i}-v_{(r)}\right)^{2}+\xi^{2}\right)\right] .
\end{aligned}
$$

The intermediate state is reconstructed from the left and right states:

$$
w_{(c)}=\int_{u<0} h^{l} f_{(l)}^{e q} \psi d \Xi+\int_{u>0} h^{r} f_{(r)}^{e q} \psi d \Xi,
$$

where $h^{l}=H(u), h^{r}=1-H(u)$ and $H$ is the Heaviside function. A third MaxwellBoltzmann distribution is then defined on the basis of $w_{(c)}$ :

$$
f_{(c)}^{e q}=\rho_{(r)}\left(\frac{\lambda_{(r)}}{\pi}\right)^{\frac{N+2}{N}} \exp \left[-\lambda_{(c)}\left(\left(u_{i}-v_{(c)_{i}}\right)^{2}+\xi^{2}\right)\right] .
$$

The distribution functions $f_{0}$ and $f^{e q}$ in Eq. (2.11) can be defined on the basis of these three states. The initial condition $f^{B G K}(x, v, 0)=f_{0}$ is defined as a solution to the BGK model (2.1) at Navier-Stokes level. $f_{0}$ is obtained from Eq. (2.10), imposing a discontinuity between left and right states and truncating the Chapman-Enskog expansion at the second term as in Eq. (2.10):

$$
f_{0}= \begin{cases}f_{(l)}^{e q}\left(\left(1+a_{(l)} x\right)-\tau\left(a_{(l)} u+A_{(l)}\right)\right), & x \leq 0, \\ f_{(r)}^{e q}\left(\left(1+a_{(r)} x\right)-\tau\left(a_{(r)} u+A_{(r)}\right)\right), & x>0,\end{cases}
$$


where $a_{(l)}$ and $a_{(r)}$ are the coefficients of spatial expansion in the phase space, $A_{(l)}$ and $A_{(r)}$ are the first order coefficient of the temporal expansions. The coefficients $a_{(l)}$ and $a_{(r)}$ may be calculated from the gradients of the conservative variables. Although it is not shown here, $a_{(l)}$ and $a_{(r)}$ are not constant values but approximated as linear functions of all degrees of freedom of the gas (microscopic velocities $u_{i}$ and internal effective degrees of freedom $\xi)$. The coefficients $A_{(l)}$ and $A_{(r)}$ are not calculated from the past history of the flow, but from the compatibility condition $\int\left(f^{e q}-f\right) \psi d \Xi=0$ at $t=0$.

The equilibrium distribution approach by $f^{B G K}$ in Eq. (2.11) is expressed as:

$$
f^{e q}= \begin{cases}f_{(c)}^{e q}\left(1+\bar{a}_{(l)} x-\bar{A} t\right), & x \leq 0, \\ f_{(c)}^{e q}\left(1+\bar{a}_{(r)} x-\bar{A} t\right), & x>0,\end{cases}
$$

where the coefficient $\bar{a}_{(l)}$ and $\bar{a}_{(r)}$ are obtained from fictitious gradients from the linear interpolation between $w_{(l)}, w_{(c)}$ and $w_{(r)}$. $\bar{A}$ are obtained from the compatibility condition integrated in a time interval.

The substitution of Eqs. (2.16) and (2.17) into Eq. (2.11) finally provides the solution to the BGK equation $f^{B G K}$ :

$$
\begin{aligned}
f^{B G K}=[ & \left.\left(1-e^{-t / \tau}\right)+u\left(-\tau+\tau e^{-t / \tau}+t e^{-t / \tau}\right)\left(h_{(l)} \bar{a}_{(l)}+h_{(r)} \bar{a}_{(r)}\right)+\left(t-\tau+\tau e^{-t / \tau}\right) \bar{A}\right] f_{(c)}^{e q} \\
& +e^{-t / \tau}\left[h_{(l)} f_{(l)}^{e q}+h_{(r)} f_{(r)}^{e q}-u(t+\tau)\left(a_{(l)} h_{(l)} f_{(l)}^{e q}+a_{(r)}^{r} h_{(r)} f_{(r)}^{e q}\right)\right] \\
& -\tau e^{-t / \tau}\left(A_{(l)} h_{(r)} f_{(l)}^{e q}+A_{(r)} h_{(r)} f_{(r)}^{e q}\right) .
\end{aligned}
$$

In order to obtain a more compact formulation, the following distribution functions at Navier-Stokes level are introduced:

$$
\begin{aligned}
& f_{(c)}=f_{(c)}^{e q}\left(1-\tau\left(h_{(l)} \bar{a}_{(l)} u+h_{(r)} \bar{a}_{(r)} u+\bar{A}\right)\right), \\
& f_{(u)}=h_{(l)} f_{(l)}^{e q}\left[1-\tau\left(a_{(l)} u+A_{(r)}\right)\right]+h_{(r)} f_{(r)}^{e q}\left[1-\tau\left(a_{(r)} u+A_{(r)}\right)\right] .
\end{aligned}
$$

$f_{(c)}$ is built from the fictitious state introduced with Eq. (2.14) and, by analogy with the terminology used for numerical schemes, may be considered central. $f_{(u)}$ keeps into account the left and right reconstructed variables, and may be related to the idea of upwind. The use of the terms central and upwind do not imply any analogy with conventional schemes involving a discontinuous reconstruction, where upwind in included to generate dissipation. When combined with Eqs. (2.19) and (2.20), Eq. (2.18) can be re-expressed:

$$
f^{B G K}=f_{(c)}(1+\bar{A} t)+e^{-t / \tau}\left(f_{(u)}-f_{(c)}\right)+t e^{-t / \tau}\left(\widetilde{f_{(u)}}-\widetilde{f_{(c)}}\right),
$$

where $\widetilde{f_{(c)}}$ and $\widetilde{f_{(u)}}$ only retain the spatial expansion coefficients:

$$
\begin{aligned}
& \widetilde{f_{(c)}}=f_{(i)}^{e q}\left(1-\tau\left(h_{(l)} \bar{a}_{(l)} u+h_{(r)} \bar{a}_{(r)} u\right)\right), \\
& \widetilde{f_{(u)}}=h_{(l)} f_{(l)}^{e q}\left[1-\tau\left(a_{(l)} u\right)\right]+h_{(r)} f_{(r)}^{e q}\left[1-\tau\left(a_{(r)} u\right)\right] .
\end{aligned}
$$


Eq. (2.21) reveals a combination of central and upwind distribution functions, whose coefficients depend on collision rate $\tau$ and time. The limit of Eq. (2.21) for a vanishing $\epsilon$ (or hydrodynamic limit) is:

$$
\lim _{\epsilon \mapsto 0} f^{B G K}=f_{(c)}(1+\bar{A} t) .
$$

Eq. (2.24) suggests that the gas-kinetic scheme for small values of $\epsilon$ generates time-accurate Navier-Stokes fluxes by means of $f_{(c)}$. Non negligible values of $\epsilon$ trigger corrections depending on reconstruction values and the degree of rarefaction $\epsilon$. This implies the capability to generate physically consistent dissipation as a reaction to a discontinuity in the reconstruction:

$$
f_{\text {corr }}^{B G K}=e^{-t / \tau}\left(f_{(u)}-f_{(c)}\right)+t e^{-t / \tau}\left(\widetilde{f_{(u)}}-\widetilde{f_{(c)}}\right) .
$$

Eq. (2.21) can then be re-expressed as:

$$
f^{B G K}=f^{N S}+f_{\text {corr }}^{B G K}(\epsilon) .
$$

It is interesting to note that the correction terms generate viscous stresses which are in principle not linearly related to the strain tensor. The stress tensor can be evaluated by integrating the fluctuations [31]:

$$
\sigma_{i j}^{B G K}=\int\left(u_{i}-v_{i}\right)\left(u_{j}-v_{j}\right) f^{B G K} d \Xi .
$$

One can now decompose the stress tensor into a Navier-Stokes component and a correction term:

$$
\sigma_{i j}^{B G K}=\sigma_{i j}^{N S}+\sigma_{c o r r}^{B G K}{ }_{i j}=\int\left(u_{i}-v_{i}\right)\left(u_{j}-v_{j}\right) f^{N S} d \Xi+\int\left(u_{i}-v_{i}\right)\left(u_{j}-v_{j}\right) f_{c o r r}^{B G K} d \Xi .
$$

It can be easily demonstrated $(\mathrm{Xu}[31])$ that $f^{N S}$ generates the "conventional" NavierStokes stress tensor:

$$
\sigma_{i j}^{N S}=-p \delta_{i j}+\mu\left(S_{(c)_{i j}}-\frac{2}{3} S_{(c)_{k k}} \delta_{i j}\right)
$$

where

$$
S_{(c) i j}=\frac{1}{2}\left(\frac{\partial v_{(c)}}{\partial x_{j}}+\frac{\partial v_{(c)}}{\partial x_{i}}\right)
$$

is the strain rate tensor and $\mu=\tau / p$. The suffix $(c)$ refers to the intermediate gas state. Interestingly, $\sigma_{\text {corr }}^{B G K}{ }_{i j}$ does not show any proportionality to a single strain tensor, as $f_{(u)}$ cannot be related to a single macroscopic state.

\subsubsection{Evaluation of fluxes in a time interval}

The solution of the BGK equation Eq. (2.21) allows the recovery of the numerical fluxes in direction $n$ in a given time interval $[0, T]$ by a simple time integration:

$$
\mathcal{F}_{n}=\int_{0}^{T} \int f^{B G K} \psi v_{n} d \Xi d t
$$


In the original scheme by $\mathrm{Xu}$ [32], the upper limit of the time interval, $T$, corresponds to the time step $T=\Delta t$. This choice may be acceptable for laminar flow, where the relaxation time $\tau$ is often much smaller than $\Delta t$, but may lead to numerical difficulties and griddependent results in turbulent flow, where the relaxation time can assume higher values.

In order to remove the dependence on the grid when using local time stepping, in this study a different upper limit $\widehat{\tau}$ has been introduced, as the local timescale of resolved flow. A common practice in rarefied flow dynamics is to evaluate $\widehat{\tau}$ on the basis of the resolved flow gradients; the gradients of density are typically used:

$$
\widehat{\tau}=\frac{\rho}{D \rho} .
$$

Time integration of Eq. (2.31) is now performed in the interval $[0, \widehat{\tau}]$ :

$$
\mathcal{F}_{n}=\int_{0}^{\widehat{\tau}} \int f^{B G K} \psi v_{n} d \Xi d t=\widehat{\tau} \int \widehat{f^{B G K}} \psi v_{n} d \Xi,
$$

where $\widehat{f^{B G K}}$ is the time average of $f^{B G K}$ :

$$
\begin{aligned}
\widehat{f^{B G K}}= & \frac{1}{\widehat{\tau}} \int_{0}^{\widehat{\tau}} f^{B G K} d t \\
= & f_{(c)}(1+1 / 2 \bar{A} \widehat{\tau})+\frac{\tau}{\widehat{\tau}}\left(1-e^{-\widehat{\tau} / \tau}\right)\left(f_{(u)}-f_{(c)}\right) \\
& \quad+\left[\frac{\tau}{\widehat{\tau}}\left(1-e^{-\widehat{\tau} / \tau}\right)-\frac{\tau}{\widehat{\tau}} e^{-\widehat{\tau} / \tau}\right]\left(\widetilde{f_{(u)}}-\widetilde{f_{(c)}}\right) .
\end{aligned}
$$

Introducing now the dimensionless quantities:

$$
\begin{aligned}
& \epsilon=\tau / \widehat{\tau}, \\
& \alpha(\epsilon)=\epsilon\left(1-e^{-1 / \epsilon}\right), \\
& \beta(\epsilon)=\epsilon e^{-1 / \epsilon},
\end{aligned}
$$

Eq. (2.34) can finally be re-arranged into the compact form:

$$
\widehat{f^{B G K}}=f_{(c)}(1+1 / 2 \bar{A} \widehat{\tau})+\alpha\left(f_{(u)}-f_{(c)}\right)+(\alpha-\beta)\left(\widetilde{f_{(u)}}-\widetilde{f_{(c)}}\right) .
$$

The dimensionless quantity $\epsilon$ is the ratio of unresolved time scales to the ones of the resolved flow and represent a particular measure of the degree of rarefaction. Eq. (2.38) reveals therefore a dependence of the fluxes on the degree of rarefaction.

In order to obtain numerical fluxes consistent with (2.38) over the time interval $[0, \Delta t]$, as is necessary in a practical calculation, an effective distribution function $\overline{f^{B G K}}$ is introduced:

$$
\mathcal{F}_{n}=\Delta t \int \overline{f^{B G K}} \psi v_{n} d \Xi
$$


$\overline{f^{B G K}}$ is obtained by expanding Eq. (2.33) in series and assuming a $\tau$-independent $\epsilon$ :

$$
\overline{f^{B G K}}=f_{(c)}(1+1 / 2 \bar{A} \Delta t)+\alpha\left(f_{(u)}-f_{(c)}\right)+(\alpha-\beta)\left(\widetilde{f_{(u)}}-\widetilde{f_{(c)}}\right) .
$$

The distribution function $\overline{f^{B G K}}$ in Eq. (2.40) is accurate to $\Delta t \tau$; the fluxes in Eq. (2.39) are accurate to $\Delta t^{2} \tau$.

\subsection{Modification of the original scheme to simulate turbulent flow}

\subsubsection{Evaluation of the relaxation time in a turbulent simulation}

A gas-kinetic scheme for turbulent flow is obtained assuming that Eq. (2.1) may be rewritten replacing the relaxation time $\tau$ with a relaxation time $\tau_{t}$, which keeps into account also unresolved turbulent fluctuations:

$$
\frac{\partial f}{\partial t}+(v \cdot \nabla) f=\frac{f^{e q}-f}{\tau_{t}} .
$$

In analogy with the original gas-kinetic scheme [32], $\tau_{t}$ could be trivially assessed adding to the relaxation time $\tau$ from Eq. (2.8) the effects an assumed eddy viscosity $\mu_{t}$ by setting merely:

$$
\tau_{t}=\frac{\mu+\mu_{t}}{p}
$$

However, a deeper analysis of the scheme may exploit the fact that the effect of unresolved turbulence is now expressed by a turbulent relaxation time and not by an eddy viscosity; $\tau_{t}$ can also be obtained in physically more meaningful way directly from assumed turbulent quantities like the turbulent kinetic energy $k$ and the turbulent dissipation rate $\varepsilon$. On the basis of a $k-\varepsilon$ RANS turbulence model and a systematic renormalization group procedure, Chen et al. [5] and Succi et al. [28] proposed:

$$
\tau_{t}^{k \varepsilon}=\frac{\mu}{p}+C_{\mu} \frac{k^{2} / \varepsilon}{T\left(1+\eta^{2}\right)^{1 / 2}}
$$

where $C_{\mu}$ is a numerical coefficient used in the $k-\varepsilon$ model, normally fixed at a value around $0.09, k$ is turbulence kinetic energy, $\varepsilon$ is turbulence dissipation rate and $\eta=S k / \varepsilon$, $S$ is a measure of the local velocity gradient. The argument used by Chen is that $\tau_{t}^{k \varepsilon}$ in Eq. (2.43) should express the dependence of $\tau_{t}$ from the variety of unresolved time scales.

Eq. (2.43) can be adapted to other turbulence models such as the well-known $k-\omega$ turbulence model by Wilcox [30]:

$$
\tau_{t}^{k \omega}=\frac{\mu}{p}+\frac{k / \omega}{T\left(1+\eta^{2}\right)^{1 / 2}}
$$

where $\omega=\varepsilon / k$ is the turbulent specific dissipation rate. The dimensionless quantity $\epsilon$ is now assessed on the basis of $\tau_{t}^{k \omega}$ :

$$
\epsilon=\tau_{t}^{k \omega} / \widehat{\tau}
$$




\subsubsection{Solution of the BGK model with a variable $\tau$}

The use of Eq. (2.11) represents an approximation, as the relaxation time $\tau$ is in reality dependent on the flow gradients. This is not peculiar to turbulent flow if the dependence of molecular viscosity on temperature is taken into account with Sutherland's law. It might be argued that the dynamics of the relaxation time is slower than the resolved flow. The validity of these assumption remains though to be consolidated.

A similar application has been followed to tackle rarefied flow; Liao et al. [18] have used the same gas-kinetic scheme with a variable relaxation time in order to model the collisions in the transitional regime.

\subsubsection{Turbulent stress tensor}

In classical turbulence modeling the linear relation between turbulent stress and strain rate tensors is adequate for many flow classes but can lead to errors [22]. More sophisticated, and in principle also more accurate, models include non-linear components of the turbulent stress tensor. The coefficients multiplying these components are derived from assumptions on the nature of turbulence and are not general. The turbulent gaskinetic scheme does not make any assumptions on the type of turbulence nor does it introduce a fixed structure for the turbulent stress tensor. Still it has the ability to generate a non-linear relation between $\sigma_{i j}$ and $S_{i j}$; Chen et al. have demonstrated that a second order Chapman-Enskog expansion leads to a turbulent stress tensor, whose non-linear coefficients are close to the ones of standard non-linear two-equation models [6]. The non-linear, corrections terms expressed by Eq. (2.28) meet the only requirement of being a solution of the BGK equation (Eq. (2.1)). This might be interpreted by saying that the mechanism behind the turbulent gas-kinetic scheme consists of supplying some "degrees of freedom" (in the case of Xu's scheme the left and right flow states and their gradients) and letting the underlying kinetic theory find the physically more consistent solution.

\subsection{Implementation of the turbulent gas-kinetic scheme into a finite-volume solver}

\subsubsection{Artificial dissipation}

In the presence of shocks, it might necessary to include artificial dissipation, through an additional relaxation time component. The relaxation time in Eq. (2.44) is modified with the addition of a contribution $\left(\tau_{a}\right)$ which provides additional dissipation in the proximity of discontinuities

$$
\tau_{t}^{k \omega}=\frac{\mu}{p}+\frac{k / \omega}{T\left(1+\eta^{2}\right)^{1 / 2}}+\tau_{a}
$$

The artificial dissipation time $\tau_{a}$, following $\mathrm{Xu}$ [32], is proportional to the pressure jump across the interface:

$$
\tau_{a}=C_{a} \frac{\left|p^{r}-p^{l}\right|}{\left|p^{r}+p^{l}\right|} \Delta t
$$


where $p^{l}$ and $p^{r}$ are pressure values of the left and right states of the gas, $C_{a}$ is a coefficient. $C_{a}$ plays a role in laminar simulations by providing numerical stability in the presence of strong shocks. Its value is often set in the $[0,1]$ range, depending on grid and reconstruction technique. In turbulent simulations its role is often less critical for numerical stability of the simulation although it contributes to stabilizing the shock.

\subsubsection{Unity Prandtl number}

A known drawback of the BGK model Eq. (2.1) is that it generates a unity Prandtl number. Complying with the original formulation of the scheme by $\mathrm{Xu}$ [32], the heath flux must be corrected to account for a realistic Prandtl number.

\subsubsection{Multidimensional implementation}

In this section, the distribution function is expanded only in the direction $x$, normal to the interface, i.e. with the coefficients $a_{(l)}, a_{(r)}, \bar{a}_{(l)}$ and $\bar{a}_{(r)}$. This choice is computationally efficient and has also been made by other researchers [20,32]. However, the scheme can be easily modified in order to include a multi-dimensional expansion. Additional coefficients are needed for the other coordinate directions; for instance Eq. (2.16) would be re-expressed as follows:

$$
f_{0}= \begin{cases}f_{(l)}^{e q}\left(\left(1+a_{(l)} x+b_{(l)} y\right)-\tau\left(a_{(l)} u_{1}+b_{(l)} u_{2}+A_{(l)}\right)\right), & x \leq 0, \\ f_{(r)}^{e q}\left(\left(1+a_{(r)} x+b_{(r)} y\right)-\tau\left(a_{(r)} u_{1}+b_{(r)} u_{2}+A_{(r)}\right)\right), & x>0 .\end{cases}
$$

A multi-dimensional version was also developed; on reasonably good-quality grids it provides comparable results at a slightly higher computational cost. On lower quality grids, the multi-dimensional version is prone to develop numerical instabilities.

\subsubsection{Allied turbulence model}

Due to its higher suitability to the chosen flow cases, the well-known $k-\omega$ turbulence model [30] has been chosen. It supplies the turbulent kinetic $k$ and turbulent specific dissipation $\omega$. The equations for $k$ and $\omega$ are solved alongside the equations for the conservative variables.

\subsubsection{The complete turbulent gas-kinetic scheme}

A turbulent gas-kinetic scheme based on the $k-\omega$ model is represented by the following set of equations:

$$
\begin{aligned}
& \epsilon^{k \omega}=\left(\frac{\mu}{p}+C_{a} \frac{\left|p^{r}-p^{l}\right|}{\left|p^{r}+p^{l}\right|} \Delta t+\frac{k / \omega}{T\left(1+\eta^{2}\right)^{1 / 2}}\right)(\rho / D \rho)^{-1}, \\
& \alpha^{k \omega}=\epsilon^{k \omega}\left(1-e^{-1 / \epsilon^{k \omega}}\right), \quad \beta^{k \omega}=\epsilon^{k \omega} e^{-1 / \epsilon^{k \omega}}, \\
& \overline{f^{k \omega}}=f^{(c)}(1+1 / 2 \bar{A} \Delta t)+\alpha^{k \omega}\left(f^{(u)}-f^{(c)}\right)+\left(\alpha^{k \omega}-\beta^{k \omega}\right)\left(\widetilde{f^{(u)}}-\widetilde{f^{(c)}}\right),
\end{aligned}
$$




$$
\mathcal{F}_{n}=\Delta t \int \overline{f^{k \omega}} \psi v_{n} d \Xi
$$

\subsubsection{Time advancing}

In the numerical experiments carried out in this work, the gas-kinetic fluxes have been implemented in a 2D finite volume steady-state solver $[23,24]$. Well-known acceleration techniques (4-level multigrid and LU-SGS preconditioning in the form proposed by Jameson et al. $[15,37])$ have provided convergence properties comparable to more traditional Navier-Stokes schemes.

\subsubsection{Variable reconstruction and limiting process}

The reconstruction techniques include second and third order TVD/MUSCL schemes and fifth order WENO, although the results shown are only second-order. The minmod limiter has been used in all cases for both conservative variables and their gradients.

\subsubsection{Boundary conditions}

No-slip wall boundary conditions have been used in all flow case; as no hypersonic cases have been simulated so far no changes with respect to conventional CFD have been implemented, as the "rarefaction" effects introduced in the previous sections are not expected to extend to solid walls.

\subsubsection{Computational costs}

With the direction-splitting turbulent gas-kinetic scheme, the execution of one iteration including a single evaluation of the numerical fluxes requires approximately $65 \%$ additional time with respect to a conventional scheme. The multidimensional gas-kinetic scheme is about twice as expensive as Navier-Stokes. There are a number of considerations to be taken into account:

- These times refer to the current implementation, which has not been optimized for speed, Xuan et al. [36] have recently developed a much faster version of the gaskinetic scheme.

- This implementation uses approximate factorization and evaluates the fluxes only once per iteration. Fully explicit conventional schemes may require multiple evaluations whereas explicit gas-kinetic schemes may still require one due to the timeaccuracy in Eq. (2.40).

- As far as time-accurate simulations are concerned, the gas-kinetic scheme may often remain stable with a larger CFL (in airfoil-flow cases the gas-kinetic scheme works at its best with CFL around 8 to 10 whereas the conventional scheme remains in the 4 to 6 range).

- As far as steady-state simulations are concerned, gas-kinetic schemes and NavierStokes schemes do not require the same amount of iterations to reach a given convergence level: in simulations without secondary flows the conventional scheme 
requires a lower number of iterations. In simulations with secondary flows (e.g. separated regions) the conventional scheme may require a higher number of iterations or not converge at all; besides it is possible that the two schemes converge to different solutions, making the comparison of computational times pointless.

\section{Numerical experiments}

\subsection{Selection of the flow cases}

The interaction between a shock wave and a boundary layer originated by a compression corner, an impinging shock or an obstacle in a duct, develops into a multi-region flow each characterized by a different complex phenomenon (a detailed discussion of the flow physics involved can be found in the rich literature available $[1,3,9,11])$. In particular, the adverse pressure gradients first causes the thickening of the boundary layer leading to a separation and to a large recirculation region. Turbulent stresses increase significantly downstream the shock wave originating a highly turbulent mixing layer in correspondence of the slip line.

The flow cases have been chosen in order to evaluate the ability of the gas-kinetic scheme to cope with these different flow aspects and predict position and size of the interaction. Moreover the relatively rich set of experimental results available, including empirical scaling laws, allows evaluating the sensitivity to Reynolds number and geometric parameters. The analysis of the results is based on the well known AGARDograph by Delery et al. [9]. The review paper by Edwards [12] was also very useful.

Beside the well-known Délery bump channel flow, three different cases of compression corner, at different angles, Reynolds and Mach number, and two cases of impinging shock have been chosen. Two of the compression corner cases concern two experiments conducted at Princeton: the well-known measurements by Settles [27], conducted at a comparatively high Reynolds number, and the more recent ones by Bookey [3], conducted at a much lower Reynolds number, to allow the comparison with DNS or LES numerical experiments. The third compression corner concerns the experiments carried out by Dolling et al. [10] on a $28^{\circ}$ ramp at Mach 5. The impinging shock cases refer to the experiments by Bookey et al. [3] and by Dupont et al. [11]. The interaction is in this case very similar to the one caused by a compression corner with an angle $2 \alpha$, when impinging shock is generated by a ramp of angle $\alpha$ [9].

It is well-accepted that the predictions obtained with RANS methods for these flow cases are generally inaccurate for one or more of the aspects mentioned above. Whereas algebraic stresses turbulence models provide better results than standard, linear twoequation models, the upstream influence distance (i.e. the distance between the corner and the point where the effect of the shock is felt by the flow) as well as the extension of the separated flow are often underpredicted; results obtained with conventional schemes can be found in $[12,14,29]$ and references therein, an excellent review can be found in the book by Babinsky and Harvey [1]. In practice, predictions in good agreements with 
Table 1: Summary of flow cases.

\begin{tabular}{||llr||}
\hline Case & Reynolds $^{1}$ & Mach $^{2}$ \\
\hline 1. Délery bump channel (Case C) [8] & $R e_{h}=1000000$ & 0.615 \\
2. Supersonic compression corner $\alpha=8^{\circ}, 16^{\circ}, 20^{\circ}, 24^{\circ}[26]$ & $R e_{\theta}=23000$ & 2.85 \\
3. Supersonic compression corner $\alpha=24^{\circ}[3]$ & $R e_{\theta}=2400$ & 2.90 \\
4. Impinging shock $\alpha=12^{\circ}[3]$ & $R e_{\theta}=2400$ & 2.90 \\
5. Impinging shock $\alpha=7^{\circ}, 8^{\circ}, 8.8^{\circ}, 9.5^{\circ}[11]$ & $R e_{\theta}=6900$ & 2.3 \\
6. Supersonic compression corner $\alpha=28^{\circ}[10]$ & $R e_{\delta}=877000$ & 4.95 \\
\hline${ }^{1} h=$ bump height, $\theta=$ momentum thickness, $\delta=$ displacement thickness; flow cases 2 and 4 also \\
include a sensitivity analysis to Reynolds number. \\
${ }^{2}$ Délery bump channel: freestream Mach $=0.615$, the flow reaches $M \simeq 1.45$ before the shock.
\end{tabular}

experiments are obtained only with unsteady approaches such as DNS and LES [12,13, 21].

\subsection{Grid independence of results}

Grid-independence is one of the objectives pursued by the modification of the gas-kinetic scheme. Results obtained with a coarse, a medium and a fine grid are shown at least for each type of flow (bump channel, compression corner, impinging shock). Flow cases 2 and 3 have been also simulated on two grids, different in size and generated with different algorithms. All computational meshes are stretched to improve shock and boundary layer resolution, the latter is guaranteed by the placement of the first layer of cells within the laminar sublayer.

\subsection{Solvers used in the simulations}

All simulations shown in this paper have been obtained with a direction-splitting gaskinetic gas using a second-order reconstruction. The transonic flow cases have also been calculated with a conventional Navier-Stokes solver, based on the same software programme with different fluxes, using a second-order reconstruction as well. Results from a conventional scheme are not proposed for the supersonic cases; since the results become for these flows particularly dependent on numerical details. However, being the flow cases very popular the reader is referred to the literature for a comparison.

\subsection{Flow cases}

\subsubsection{Flow Case 1. Délery bump channel}

The experiment transonic bump flow (Case C), experimentally investigated by Délery [8] was designed to produce a strong shock-boundary layer interaction leading to a large flow separation. The Mach 0.615 duct flow impacts a ramp-semicircular bump mounted 

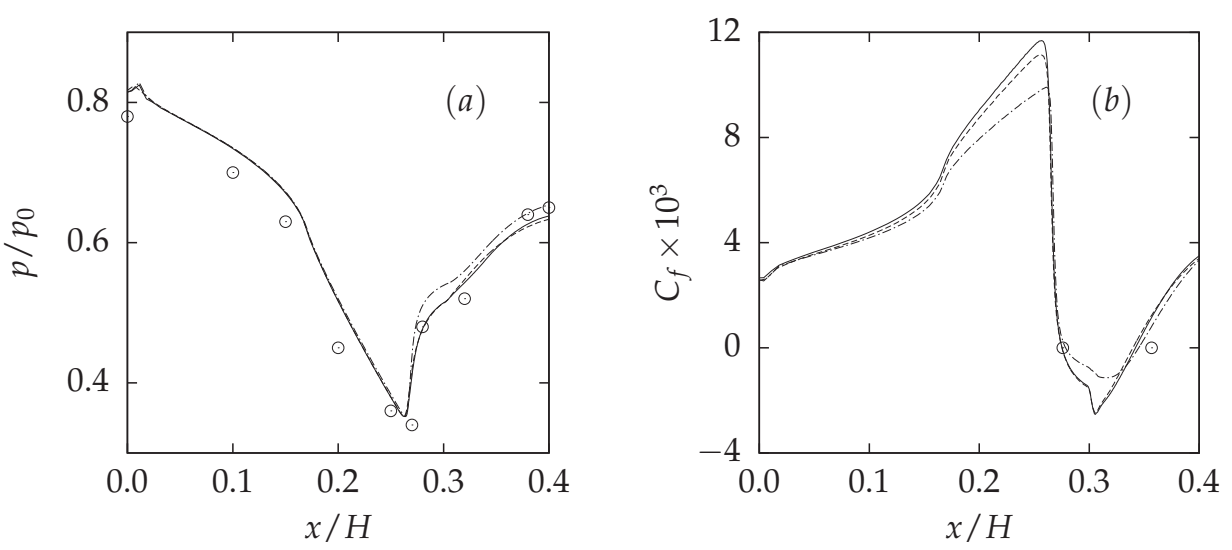

Figure 1: Pressure (a) and skin friction coefficient (b) for the Délery bump channel flow. ( -) Gas-kinetic scheme (GKS) on finest grid, (----) GKS on medium grid, (-----) GKS on coarsest grid, (-- - NavierStokes (Roe's approximate Riemann solver) on finest grid, ( o ): experimental data from Délery [8]. Size of coarse, medium and fine grids: $200 \times 160,312 \times 256,456 \times 288$ respectively.

on one side of the channel, reaching approximately Mach 1.45 before the shock. The shock-boundary layer interaction generates the typical $\lambda$ structure, with the separation starting at the foot of the first leg. It is well known that predicting the position of the separation point and the size of the separated area are challenging for standard, linear two-equation models as they tend to delay separation and underpredict the extension of the separation.

In order to match the experimental position of the shock, the outlet pressure is heuristically adjusted. The two solvers therefore use slightly different values of outlet pressure. The calculation is fully turbulent.

Figs. 1 to 4 show the results of a simulation conducted with the turbulent gas-kinetic scheme and results obtained from the same solver but with a conventional Navier-Stokes scheme with Roe's approximate Riemann solver. Fig. 1 shows static pressure and skin friction coefficient. The predicted extension of the separation region is in good agreement with experimental data for both schemes, but the behavior of static pressure downstream of the shock is more accurately predicted by the gas-kinetic scheme. The predicted shock structure, shown in Fig. 3, is different between the two schemes. One way to interpret this result, is that where the conventional scheme simply identifies a discontinuity, the gas-kinetic scheme tries to resolve it in a physically meaningful way.

Fig. 4 shows the distribution of turbulent stress $\tau_{x y}$. The agreement with experimental values (e.g. the PIV investigation by Sartor [25], not shown here) is only qualitatively acceptable (the maxima are about $20 \%$ apart).

The degree of rarefaction is shown in Fig. 2: high values, in the same order as the ones observed in the airfoil flow cases, appear in the proximity of the shocks and might be at the origin of the different behavior of the two schemes. 


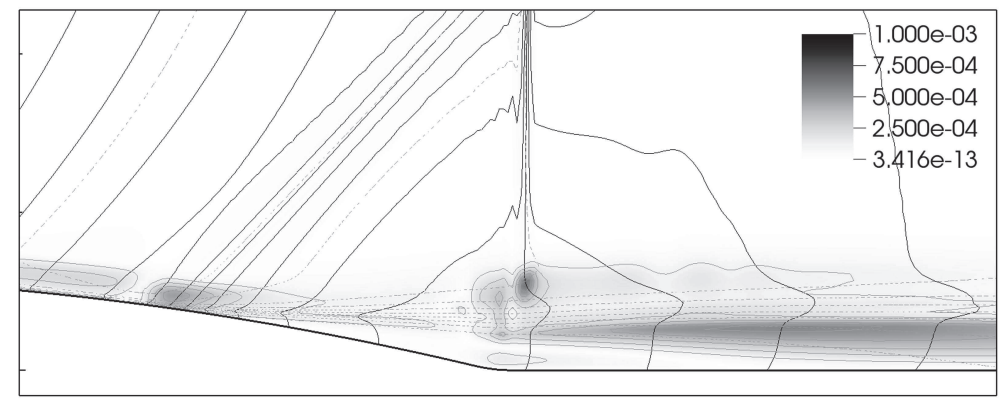

Figure 2: Degree of rarefaction according to Eq. (2.49) (iso-contours in grayscale) for Délery bump channel flow. 20 pressure isocurves have been added for reference.
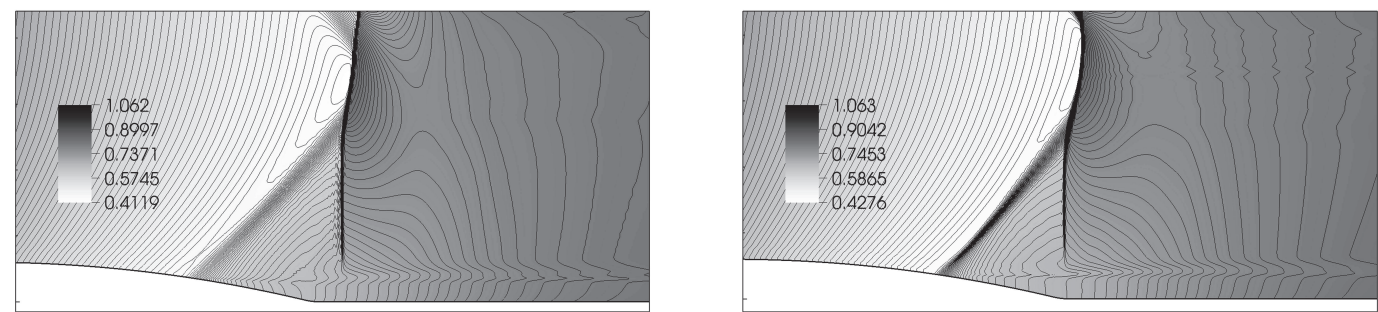

Figure 3: Flow Case 1. Sketches of the shock system in Délery bump channel flow. Gas-kinetic scheme on the left, Navier-Stokes on the right. In both figures, pressure is represented by iso-contours in grayscale. 100 pressure contour lines have also been added.

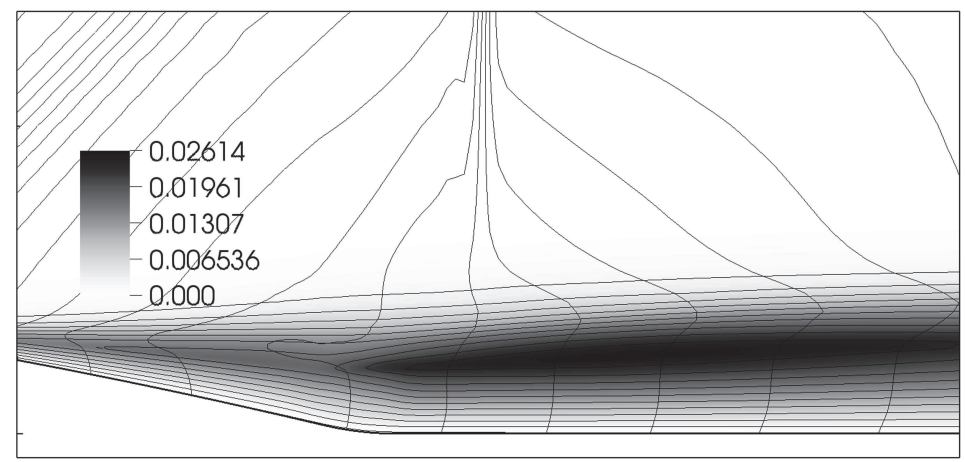

Figure 4: Flow Case 1. Non-dimensional $\tau_{x y}$ component of the turbulent stress tensor in Délery bump channel flow. Experimental values can for instance be found in the measurements by Sartor et al. [25].

\subsubsection{Flow Case 2. Supersonic compression corner at high Reynolds number}

This flow case concerns the well known experiments carried out by Settles [27]. The experiments include four ramp angles: $8^{\circ}, 16^{\circ}, 20^{\circ}$ and $24^{\circ}$. The smallest angle is not sufficient to separate the boundary layer, the $16^{\circ}$ ramp is just enough to cause an incipient 

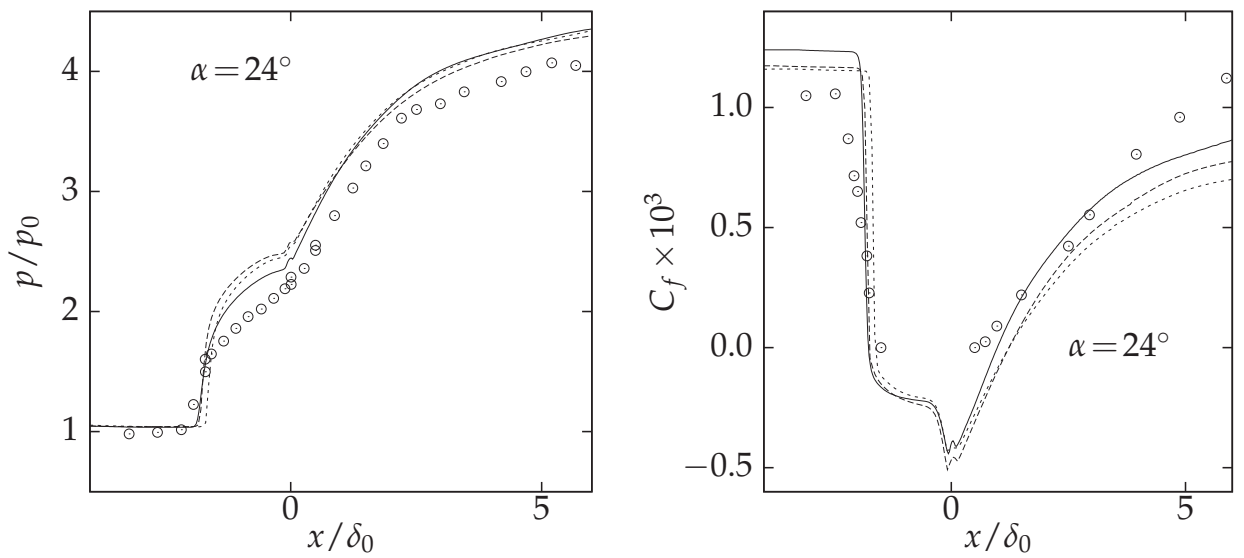

Figure 5: Flow Case 2. Grid convergence. (freestream conditions: $M=2.85, R e=7.0 \times 10^{7}$ per length unit, $\left.\delta_{0}=0.023 \mathrm{~m}\right)$. ( ( $)$ Gas-kinetic scheme (GKS) on finest grid, (---- ) GKS on medium grid (only shown for $\left.\alpha=24^{\circ}\right),(----)$ GKS on coarsest grid (only shown for $\left.\alpha=24^{\circ}\right),(\circ)$ : experimental data from Settles [26].
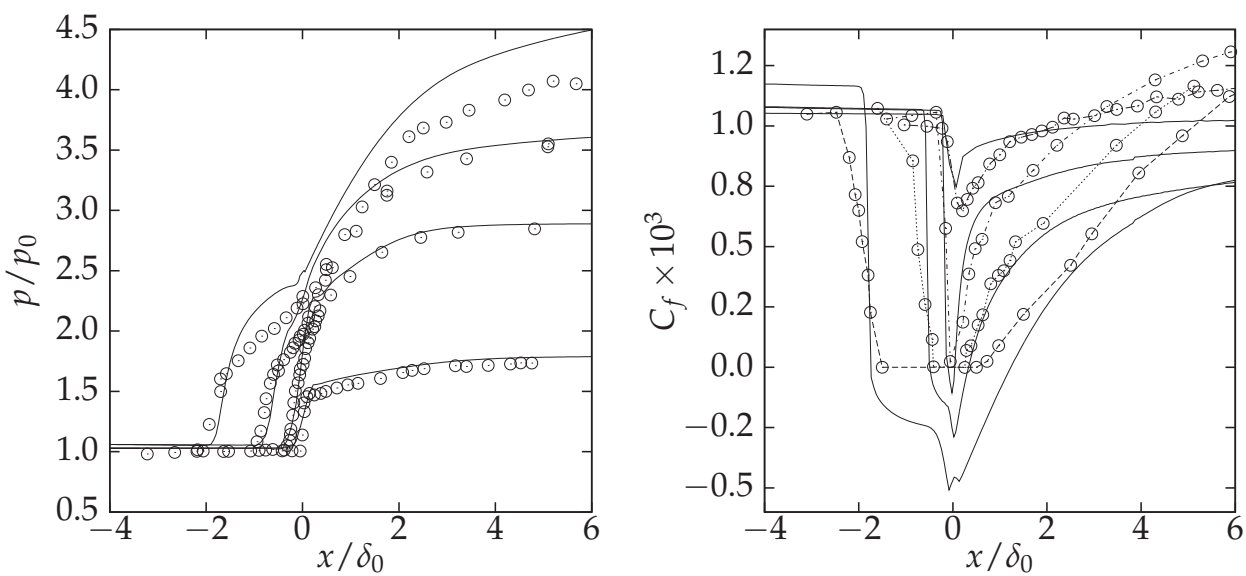

Figure 6: Flow Case 2. Pressure and friction coefficient calculated for four different compression corner flows, with angles of $8^{\circ}, 16^{\circ}, 20^{\circ}$ and $24^{\circ}$ (freestream conditions: $M=2.85, R e=7.0 \times 10^{7}$ per length unit, $\delta_{0}=0.023 \mathrm{~m}$ ). $(-)$ GKS on finest grid, ( o ): experimental data from Settles [26].

separation, whereas separation occurs with the two steeper angles. Whereas conventional schemes seem able to simulate the first two cases (also the computations published by Settles in 1979 [26] were in good agreement with experiments), the separation area is systematically underpredicted.

Pressure and skin friction coefficient obtained with the turbulent gas-kinetic scheme for Settles' experiment are shown in Fig. 6. The evidence of grid convergence is shown in Fig. 5 for the $24^{\circ}$ ramp. For all ramp angles, the good agreement in the interaction area is evident whereas the flow after-reattachment is predicted with lower accuracy. A 


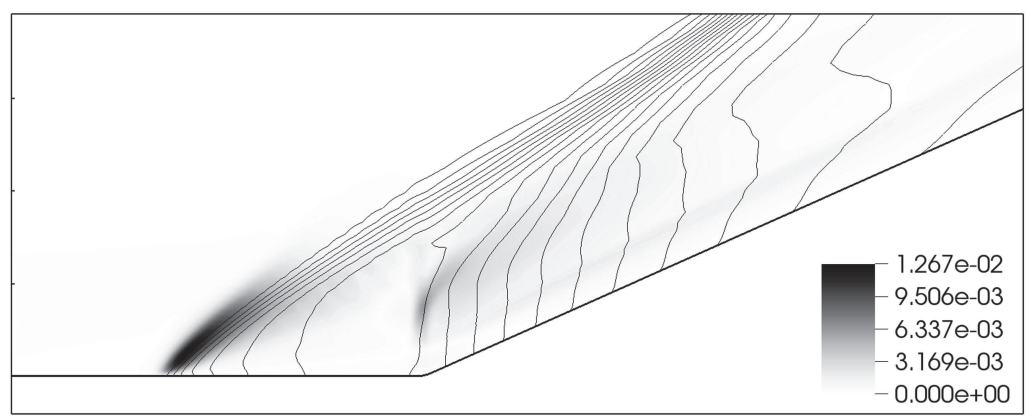

Figure 7: Flow Case $2 \alpha=24^{\circ}$. Degree of rarefaction according to Eq. (2.49) in supersonic compression corner flow, $M=2.85$ (iso-contours in grayscale). 20 pressure contour lines have been added for reference.

likely cause is related to the inadequacy of the underlying $k-\omega$ model to separated flow in supersonic regime.

A further aspect investigated in this flow case is the sensitivity to Reynolds number. In particular, the upstream interaction length has been calculated at different Reynolds numbers (for the $24^{\circ} \mathrm{ramp}$ ), the results are summarized in Table 2. Interestingly, the lengths calculated seem to fit acceptably into the empirical relation devised by Settles [9] for flows at $M \simeq 3$ :

$$
\left(\frac{L_{0}}{\delta_{0}}\right) R e_{\delta_{0}}^{1 / 3}=0.9 e^{0.23 \alpha}
$$

where $L_{0}$ is the upstream influence length and $\delta_{0}$ is the boundary layer thickness.

Table 2: Flow Case 2. Compression corner, $M=2.85, \alpha=24^{\circ}$. Sensitivity to Reynolds number. Empirical law $0.9 e^{0.23 \alpha}[9]$.

\begin{tabular}{||rrrr||}
\hline$R e_{\delta_{0}}$ & $L_{0} / \delta_{0}$ & $\left(\frac{L_{0}}{\delta_{0}}\right) R e_{\delta_{0}}^{1 / 3}$ & Error $(\%)$ \\
\hline 740,000 & 2.38 & 216.74 & 3.53 \\
$1,694,000$ & 1.98 & 236.34 & 5.19 \\
$2,120,000$ & 1.70 & 218.15 & 2.90 \\
$2,912,000$ & 1.63 & 233.42 & 3.90 \\
\hline
\end{tabular}

\subsubsection{Flow Cases 3 and 4. Supersonic compression corner and impinging shock at low Reynolds number}

Both flow cases concern the experiments carried out by Bookey [3], devised especially to provide a benchmark case for LES and DNS. The first experiment concerns a ramp with an angle of $24^{\circ}$, the second an impinging shock generated by a ramp with an angle of $12^{\circ}$.

Fig. 8 shows the pressure distributions for both the compression corner and the impinging shock, which are in reasonably good agreement with the experiments. Fig. 9 

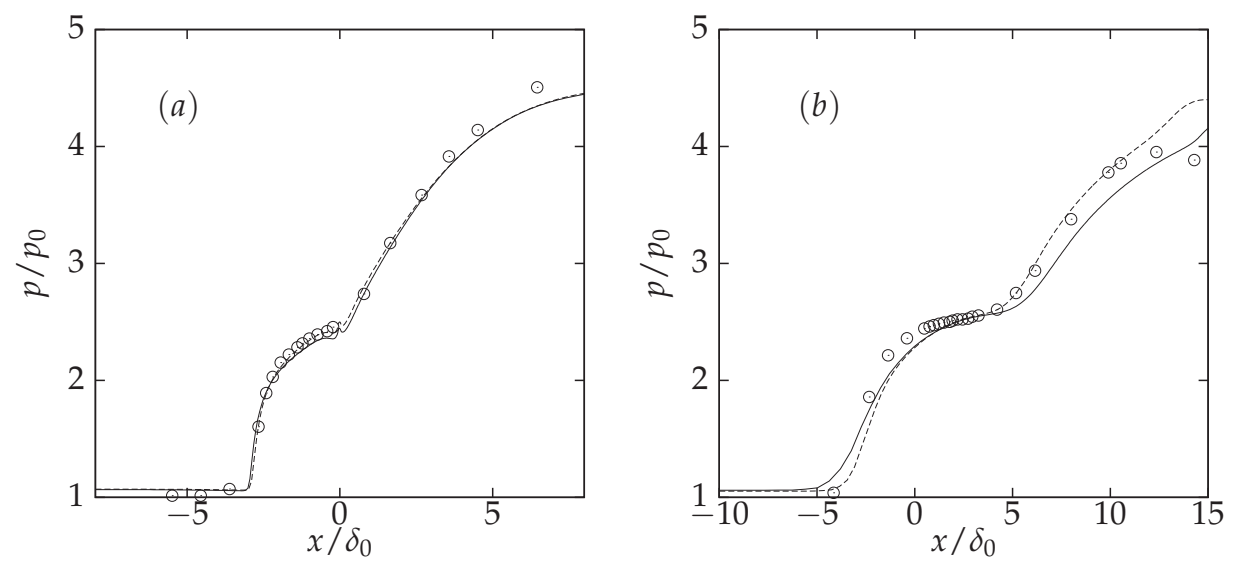

Figure 8: Flow Cases 3 and 4. Distribution of static pressure calculated for the compression corner (a) with an angle of $24^{\circ}$ and for the reflected shock (b) originating form a compression corner at $12^{\circ}$. Freestream conditions (both flows) are $M=2.90, R e_{\theta}=2400$. ( $(\longrightarrow)$ GKS on grid 1, (-----) GKS on grid 2, ( o ): experimental data from Bookey [3]. Grid sizes compression corner: $384 \times 192,512 \times 168$ respectively, grid size impinging shock: $384 \times 208,496 \times 304$ respectively. Grid 1 and 2 have different resolution and have been generated with different algorithms.

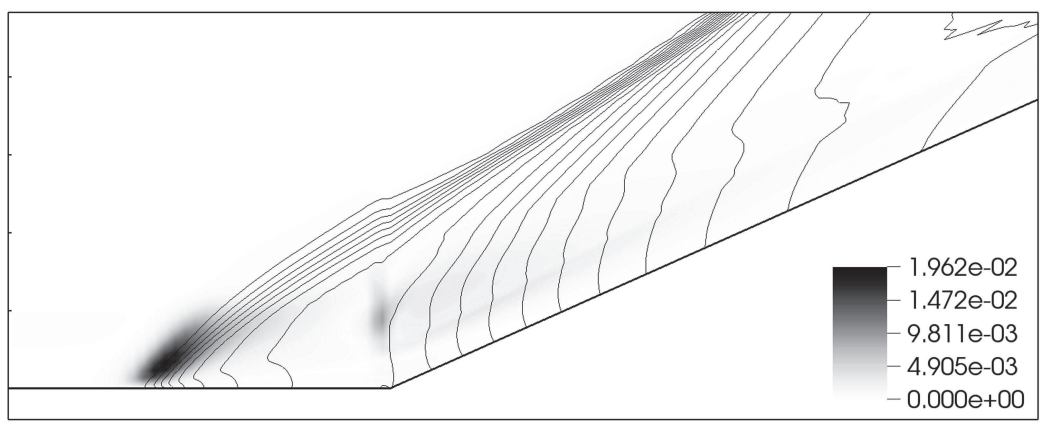

Figure 9: Degree of rarefaction according to Eq. (2.49) in Flow Case 3, supersonic compression corner, $M=2.90$ (iso-contours in grayscale). 20 pressure contour lines added for reference.

shows the distribution of the degree of rarefaction in the case of the compression corner, which reaches significant values across the shock wave and on the slip line.

Table 3 summarizes the evaluation of the upstream influence distance - in the case of the compression corner - as a function of Reynolds number, comprised in a range between 1900 and 5000. The trend is compared with Eq. (3.1), the scaling law proposed by Settles [9] used in Flow Case 2. In particular, the relation between the upstream interaction length and the (displacement thickness based) Reynolds number can be empirically related to Eq. (3.1) with the exponent changed from 0.23 to $0.1885:\left(L_{0} / \delta_{0}\right) \operatorname{Re}_{\delta_{0}}^{1 / 3}=$ $0.9 \exp (0.1885 \alpha)$. 
Table 3: Flow Case 3. Compression corner, $M=2.90, \alpha=24^{\circ}$. Upstream influence distance as a function of Reynolds number. Empirical law adjusted to $0.9 e^{0.1882 \alpha}$ (original: $0.9 e^{0.23 \alpha}$ [9]).

\begin{tabular}{||rrrr||}
\hline$R e_{\theta}$ & $L_{0} / \delta_{0}$ & $\left(\frac{L_{0}}{\delta_{0}}\right) R e_{\delta_{0}}^{1 / 3}$ & Error $(\%)$ \\
\hline 1900 & 2.70 & 80.72 & 2.03 \\
2300 & 2.58 & 81.85 & 0.65 \\
3200 & 2.35 & 83.59 & 1.45 \\
5900 & 1.92 & 84.08 & 2.05 \\
\hline
\end{tabular}

\subsubsection{Flow Case 5. Supersonic impinging shock at low Reynolds number}

The experiment by Dupont [11] is similar to flow case 3, carried at a Mach of 2.3 and a slightly different Reynolds number. It has the advantage of providing results for four different angles: $7^{\circ}, 8^{\circ}, 8.8^{\circ}$ and $9.5^{\circ}$ respectively. The pressure distribution is reported in Fig. 10 and shows a reasonably good agreement with the experiment. The degree of rarefaction, shown in Fig. 11, reaches significant values at the foot of the impinging shock and at the separation point.
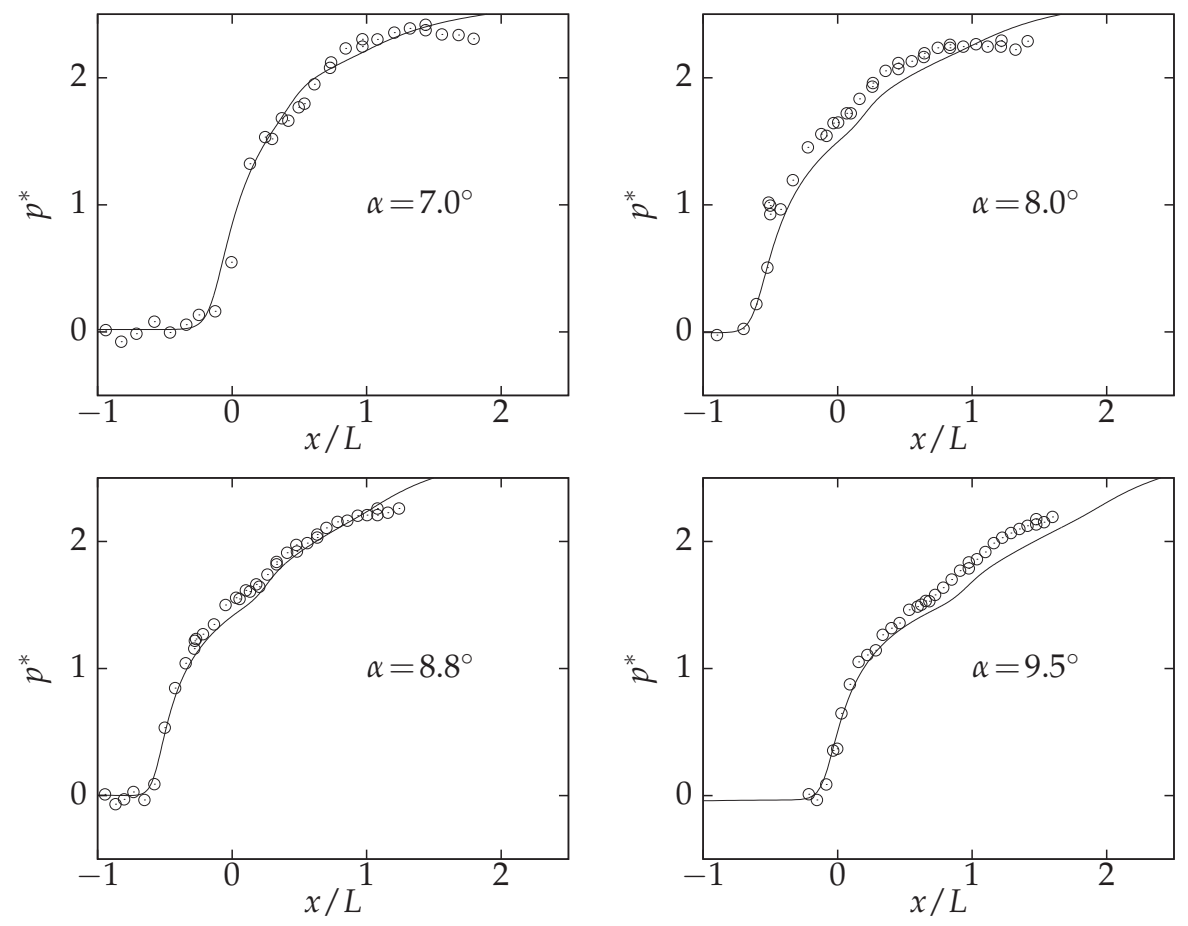

Figure 10: Flow Case 5. Reflected shock wave at low Reynolds number and Mach $M=2.3$ (experimental data from [11]) with angles ranging from 7.0 to 9.5 degrees. $p^{*}=\left(p-p_{1}\right) /\left(p_{2}-p_{1}\right)$, where $p_{1}$ and $p_{2}$ are the inviscid pressure values upstream and downstream of shock, respectively. The interaction length $L$ is measured from the foot of the reflected shock to the reattachment point. Results obtained on a grid with size: $496 \times 304$. 


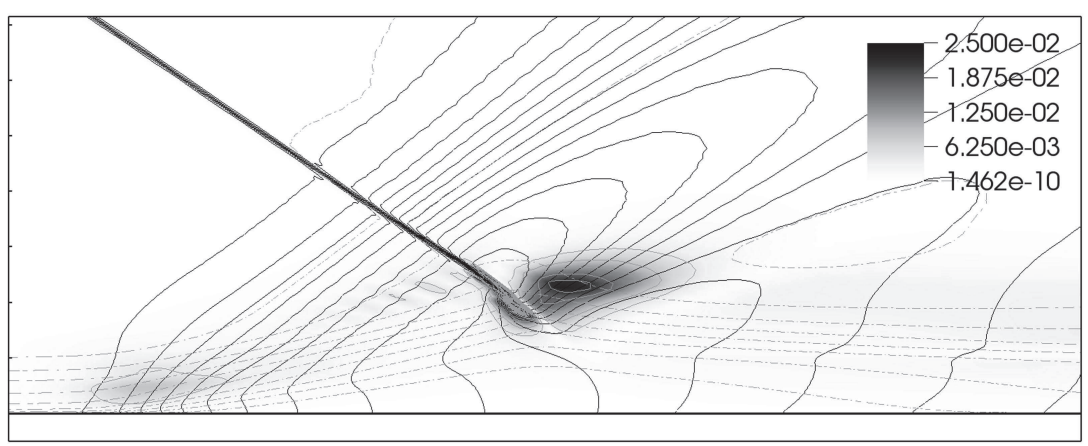

Figure 11: Degree of rarefaction according to Eq. (2.49) in Flow Case 5, reflected shock, $M=2.30$ (iso-contours in grayscale). 20 pressure contour lines have been added for reference.

\subsubsection{Flow Case 6. Supersonic compression corner at Mach 5}

This flow case, investigated by Dolling et al. [10], has been selected to extend the mach Number range to the borders of hypersonics. This flow case has been however calculated assuming adiabatic wall conditions. Results from RANS and hybrid simulations can be found in Edwards et al. [12]. In Fig. 12 the pressure distribution predicted by the turbulent gas-kinetic scheme is compared to the experimental values by Dolling et al. [10], highlighting an acceptable agreement. Fig. 13 shows the distribution of the degree of rarefaction, which reaches values as high as 0.07 .

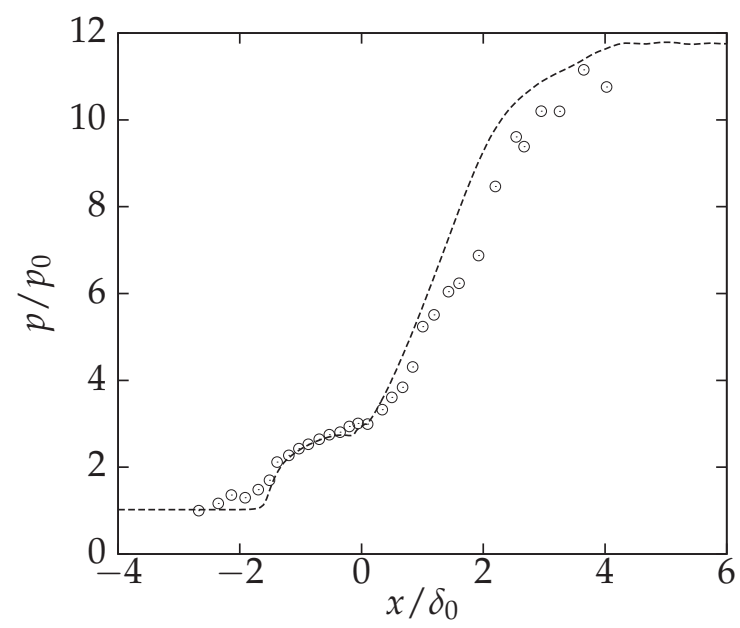

Figure 12: Compression corner $M=5$, Flow Case 6. Experimental values from Dolling et al. [10]. Results obtained on a grid with size: $384 \times 160$. 


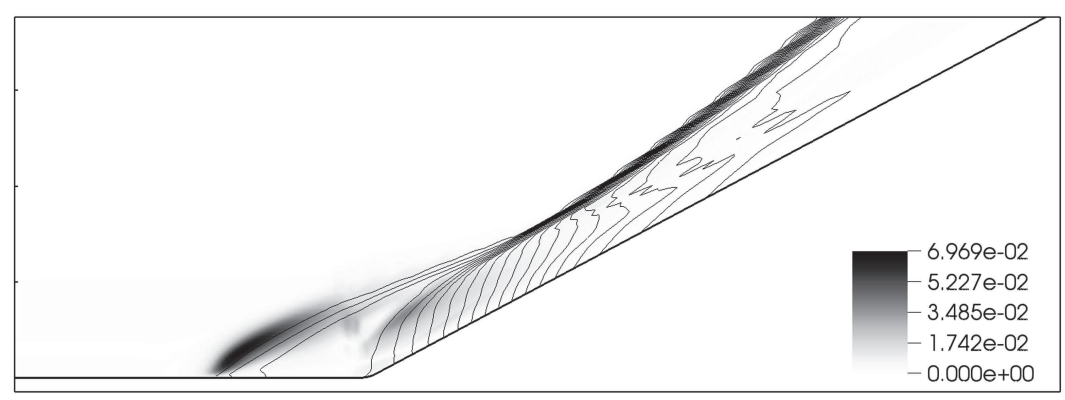

Figure 13: Degree of rarefaction according to Eq. (2.49) in Flow Case 6, supersonic compression corner, $M=4.95$ (iso-contours in grayscale). 20 pressure contour lines have been added for reference.

\section{Conclusions}

The predictions obtained with the turbulent gas-kinetic scheme are remarkably close to the experimental value, at least in terms of mean values and shock position. The turbulent gas-kinetic scheme does not affect in any way the modeling of the two turbulent quantities, $k$ and $\omega$, which are provided by a standard model. Yet, the interaction with the shock is handled, in all examples, in a much more accurate and convincing way. The degree of rarefaction, measured in terms of timescales ratio, reaches in shocklayers values well above the upper boundary of the continuous regime.It is precisely in these "rarefied" flow regions that the numerical fluxes and the converged steady-state solution provided by the turbulent gas-kinetic differ from conventional schemes. This is due to the kinetic effects generated by the underlying Boltzmann equation, i.e. to its "multiscalarity".

It is well known [1] that conventional schemes fail to systematically provide accurate predictions of the interaction between a shock and a turbulent boundary layer, in particular when it gives rise to a separated flow region. It would be difficult to provide a quantification of the error as criteria might differ from flow case to flow case and from one application to the other. For instance, the position of the shock foot, predicted by conventional schemes and expressed in percentage of the interaction length, can differ from the experimental value by $20 \%$ or more, of the overall interaction length [1]. For this particular criterion, the gas-kinetic scheme limits the error to a few percents. Additional criteria concern separation point and length of the separated flow region. The difference between the predictions obtained with conventional CFD schemes and experimental data may also in these cases be measured in a range of $10 \%$ to $20 \%$ of the overall interaction length [1] whereas the predictions by the turbulent gas-kinetic scheme presented in this paper generate errors in a much smaller range (we could estimate between a few percent to $10 \%$ of the overall interaction length). However, it would be simplistic to limit the analysis to geometric parameters: the real advantage of the turbulent gas-kinetic scheme is that it can capture effects that escape to conventional CFD. A remarkable feature in the simulations presented in this paper is possibly the resemblance of the shock structure 
with "textbook" figure [1,9]. Whereas the conventional schemes handle the shocklayer as a mere discontinuity, the gas-kinetic scheme has the ability to provide a physically meaningful solution [32] - within the limits of the available resolution. The error in the prediction of the position of the shock foot or the size of the separation bubble may be seen as an indicator revealing the weakness of the physical model. The miscalculations of heat flux in space programs - which have lead to extremely dangerous situations are notorious [1]; still conventional numerical prediction have not yet reached maturity for shock-wave interaction. Incidentally, rarefaction increases with Mach and decreases with Reynolds. Potentially, the gas-kinetic scheme seems to have the potential to improve predictions in special conditions such as those found in hypersonic flight.

The mechanisms behind the turbulent gas-kinetic scheme exploit the multiscalarity of the kinetic approach; the analysis presented in this paper reveals that the turbulent gaskinetic scheme includes a rarefaction "sensor" which activates the deviations from the conventional schemes. These corrections terms are modeled on the basis of the underlying gas kinetic theory and, unlike conventional turbulence modeling, do not require any assumption on the nature of the turbulence. Still, they represent a remarkable advantage over conventional schemes which do not dispose of any multiscale mechanisms.

The properties of turbulent gas-kinetic schemes are still largely unknown. It might be useful to investigate the influence of the main parameters such as the order of the Chapman-Enskog expansion for $f_{0}$, the reconstruction order, the time integration and the choice of the pre-conditioning. Further, validation is still at its beginnings: threedimensional flow cases are still unexplored and so is the role played by the choice of the platform model.

An extension to unsteady simulations and in particular to Large Eddy Simulation or to RANS-LES hybrid techniques is possible without any significant change to the scheme, provided a model for the subgrid turbulent relaxation time can be adapted from existing LES subgrid models. The turbulent gas-kinetic scheme might even be more suitable to LES, since, as pointed out by Chen et al. [6], the largest unresolved scale of motion is normally not much smaller than the smallest resolved scale, leading to large $\tau / \widehat{\tau}$ or $\tau / \Delta t$ ratios.

\section{References}

[1] H. Babinsky and J. K. Harvey. Shock Wave-Boundary-Layer Interactions. Cambridge University Press, New York, 2011.

[2] P.L. Bhatnagar, E.P. Gross, and M. Krook. A model for collision processes in gases. I. Small amplitude processes in charged and neutral one-component systems. Phys. Rev., 94(3):511$525,1954$.

[3] P. Bookey, C. Wyckham, and A. Smits. Experimental investigations of Mach 3 shock-wave turbulent boundary layer interactions. AIAA Paper No. 2005-4899, 2005.

[4] C. Cercignani. The Boltzmann Equation and its Applications. Springer, New York, 1988.

[5] H. Chen, S. Kandasamy, S. Orszag, R. Shock, S. Succi, and V. Yakhot. Extended Boltzmann kinetic equation for turbulent flows. Science, 301(5633):633-636, 2003. 
[6] H. Chen, S.A. Orszag, I. Staroselsky, and S. Succi. Expanded analogy between Boltzmann kinetic theory of fluids and turbulence. J. Fluid Mech., 519(1):301-314, 2004.

[7] S.Y. Chou and D. Baganoff. Kinetic flux-vector splitting for the Navier-Stokes equations. J. Comput. Phys., 130(2):217-230, 1997.

[8] J. Délery. Experimental investigation of turbulence properties in transonic shock/boundarylayer interactions. AIAA J., 21:180-185, 1983.

[9] J. Délery, J.G. Marvin, and E. Reshotko. Shock-wave boundary layer interactions. AGARDograph, 280, 1986.

[10] D. S. Dolling and M. E. Erengil. Unsteady wave structure near separation in a Mach 5 compression rampinteraction. AIAA J, 29(5):728-735, 1991.

[11] P. Dupont, C. Haddad, and J.F. Debiève. Space and time organization in a shock-induced separated boundary layer. J. Fluid Mech., 559:255-278, 2006.

[12] J. R. Edwards. Numerical simulations of shock/boundary layer interactions using timedependent modeling techniques: A survey of recent results. Prog. Aerosp. Sci., 44(6):447465, 2008.

[13] E. Garnier, N. Adams, and P. Sagaut. Large Eddy Simulation for Compressible Flows. Springer, 2009.

[14] U. Goldberg, O. Peroomian, and S. Chakravarthy. Application of the k-e-R turbulence model to wall-bounded compressive flows. AIAA Paper No. 1998-0323, 1998.

[15] A. Jameson. Solution of the Euler equations for two dimensional transonic flow by a multigrid method. Appl. Math. Comput., 13(3-4):327-356, 1983.

[16] M. N. Kogan. Rarefied gas dynamics. Plenum Press, New York, 1969.

[17] Q. Li, K. Xu, and S. Fu. A high-order gas-kinetic Navier-Stokes flow solver. J. Comput. Phys., 229(19):6715-6731, 2010.

[18] W. Liao, L. Luo, and K. Xu. Gas-kinetic scheme for continuum and near-continuum hypersonic flows. J. Spacecraft Rockets, 44(6):1232-1240, 2007.

[19] J.C. Mandal and S.M. Deshpande. Kinetic flux vector splitting for Euler equations. Comput. Fluids, 23(2):447-478, 1994.

[20] G. May, B. Srinivasan, and A. Jameson. An improved gas-kinetic BGK finite-volume method for three-dimensional transonic flow. J. Comput. Phys., 220(2):856-878, 2007.

[21] S. Pirozzoli, M. Bernardini, and F. Grasso. Direct numerical simulation of transonic shock/boundary layer interaction under conditions of incipient separation. J. Fluid Mech., 657:361-393, 2010.

[22] S.B. Pope. Turbulent Flows. Cambridge University Press, Cambridge, 2000.

[23] M. Righi. A gas-kinetic scheme for the simulation of turbulent flows. In M. Mareschal and A. Santos (Eds.), Proceeding of the 28th Internaltional Symposium on Rarefied Gas Dynamics, Zaragoza, pages 481-488. American Institute of Physics, 2012.

[24] M. Righi. A finite-volume gas-kinetic method for the solution of the Navier-Stokes equations. Royal Aeronautical Society, Aeronaut. J., (117)(1192), 2013.

[25] F. Sartor, G. Losfeld, and R. Bur. Piv study on a shock-induced separation in a transonic flow. Exp. Fluids, 53(3):815-827, 2012.

[26] G.S. Settles, T.J. Fitzpatrick, and S.M. Bogdonoff. Detailed study of attached and separated compression corner flowfields in high Reynolds number supersonic flow. AIAA J., 17(6):579-585, 1979.

[27] G.S. Settles, I.E. Vas, and S.M. Bogdonoff. Details of a shock-separated turbulent boundary layer at a compression corner. AIAA J., 14(12):1709-1715, 1976.

[28] S. Succi, O. Filippova, H. Chen, and S. Orszag. Towards a renormalized lattice Boltzmann 
equation for fluid turbulence. J. Stat. Phys., 107(1-2):261-278, 2002.

[29] S. Wallin and A.V. Johansson. An explicit algebraic Reynolds stress model for incompressible and compressible turbulent flows. J. Fluid Mech., 403:89-132, 2000.

[30] D. C. Wilcox. Turbulence Modeling for CFD, 3rd edition. DCW Industries, Inc., La Canada CA, 2006.

[31] K. Xu. Gas-kinetic schemes for unsteady compressible flow simulations. Von Karman Institute, Computational Fluid Dynamics, Annual Lecture Series, 29th, Rhode-Saint-Genese, Belgium, 1998.

[32] K. Xu. A gas-kinetic BGK scheme for the Navier-Stokes equations and its connection with artificial dissipation and Godunov method. J. Comput. Phys., 171(1):289-335, 2001.

[33] $\mathrm{K}$. Xu and J. Huang. A unified gas-kinetic scheme for continuum and rarefied flows. J. Comput. Phys., 229(20):7747-7764, 2010.

[34] K. Xu, M. Mao, and L. Tang. A multidimensional gas-kinetic BGK scheme for hypersonic viscous flow. J. Comput. Phys., 203(2):405-421, 2005.

[35] K. Xu and K.H. Prendergast. Numerical Navier-Stokes solutions from gas kinetic theory. J. Comput. Phys., 114(1):9-17, 1994.

[36] L. Xuan and K. Xu. A new gas-kinetic scheme based on analytical solutions of the BGK equation. J. Comput. Phys., 2012.

[37] S. Yoon and A. Jameson. Lower-upper symmetric-Gauss-Seidel method for the Euler and Navier-Stokes equations. AIAA J., 26(9):1025-1026, 1988. 